\title{
Management of nasal septal abscess in childhood: our experience
}

\author{
C. Dispenza*, C. Saraniti, F. Dispenza, C. Caramanna, F.A. Salzano
}

Clinica Otorinolaringoiatrica, Università di Palermo, Azienda Ospedaliera Policlinico "P. Giaccone", Via P. E. Giudici n.37, Palermo 90127, Italy

Received 18 March 2004; received in revised form 24 May 2004; accepted 26 May 2004

\section{KEYWORDS}

Nasal trauma;

Septum abscess;

Surgical treatment

\begin{abstract}
Summary
A nasal septal abscess is usually the result of an infected hematoma of the septum. A secondary septal abscess may be the result of infections extending from any of the neighbouring tissues.

The necrosis of septal cartilage may lead to nasal deformities and severe impairment of nasal patency and growth.

Objectives: Assess if the drainage of the abscess and the immediate reconstruction of the destroyed nasal septum in the acute phase is the best treatment to prevent short- and long-term effect on nasal and midface growth.

Methods: Three pediatric patients treated with drainage and immediate implantation of homologous bank cartilage prior to 1990 and four treated with mosaic plastic using small pieces of residual septal cartilage assembled with fibrin glue.

Result: No complication were observed in the follow-up and any deformities in the long-term controls.

Conclusions: The drainage and immediate reconstruction of the nesal septum are the golden standard in the treatment of the septum infected haematoma.

(C) 2004 Elsevier Ireland Ltd. All rights reserved.
\end{abstract}

\section{Introduction}

A nasal septal abscess is defined as a collection of pus between the cartilage or bony septum and the mucoperichondrium or mucoperiostium [1] (Fig. 1). The recognition of the nasal septal abscess is traced

* Corresponding author. Tel.: +390916554243.

E-mail address: francesco.dispenza@poste.it (C. Dispenza). back to 1810 when Cloquet healed an abscess by drainage [2].

This pathology is often the result of an infected septal hematoma which can be a serious complication of trauma or surgery. The rupture of the small vessels that supply the nasal septum forms a hematoma that separates the mucoperichondrium from the septal cartilage. Cartilage destruction follows as a result of ischemic and pressure necrosis. The 


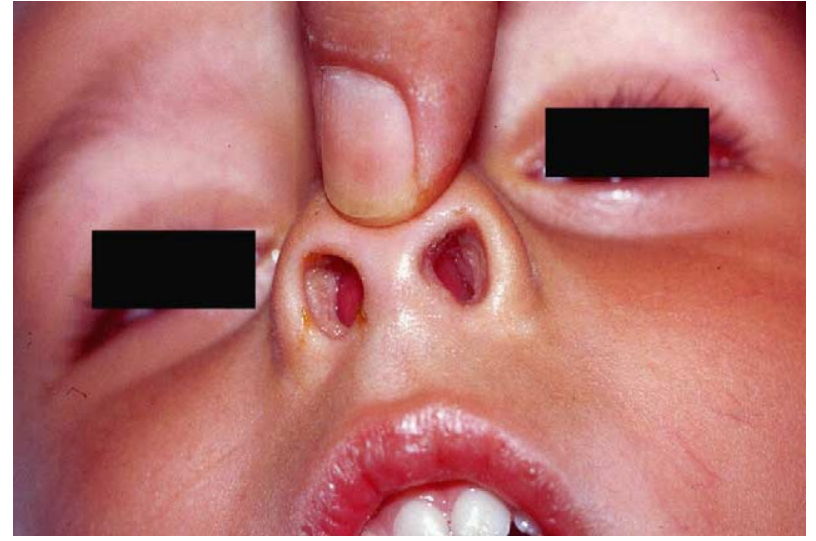

Fig. 1 A nasal septal abscess in a 2-year-old child.

static blood and the necrotic cartilage form an adequate medium for the growth of the bacteria which normally colonize the nasal mucosa [3].

Beck defines this process as a post-trauma primary abscess and groups all the other pathologies as secondary complications to the infections extending from the neighbouring tissues (dental infections, septic embolism, furuncle of the nasal vestibule); in such cases, the infection focus should be identified and treated at the same time as the nasal septal abscess (secondary abscesses) [15]. The most common organisms cultured from nasal abscesses are Staphylococcus aureus, Streptococcus pneumoniae, Haemophilus influenzae, [3-5] and group A beta-haemolytic streptococcus [1]; Brook reports two cases in which cultures for anaerobic bacteria revealed Prevotella intermedia and Peptostreptococcus magnus in one patient, and Peptostreptococcus anaerobius, Streptococcus intermedius and Prevotella melaninogenica in the second patient. Brooks believes these organisms are fairly unremarkable as they are usually cultured from oropharynx and retropharynx infections, cervical ademolymphitis and parotidean abscesses [13].

Pirsig believes the cartilage lysis is not just a consequence of the avascular necrosis but occurs for the digestion process caused by leukocytes and Cathepsin D enzyme. The latter is present in the septal cartilage and belongs to a group of enzymes which re-shape the quadrangular cartilage. Its acid $\mathrm{pH}$ accelerates the cartilage digestion [2] (Fig. 2).

Inspection with palpation is initially required to detect the destroyed cartilage. The nose undergoes a slow collapse in the weeks following the injury because of a retraction in the connective tissue, skin, mucous covering, dorsum and valve. The short-term effects of the septal abscess are the

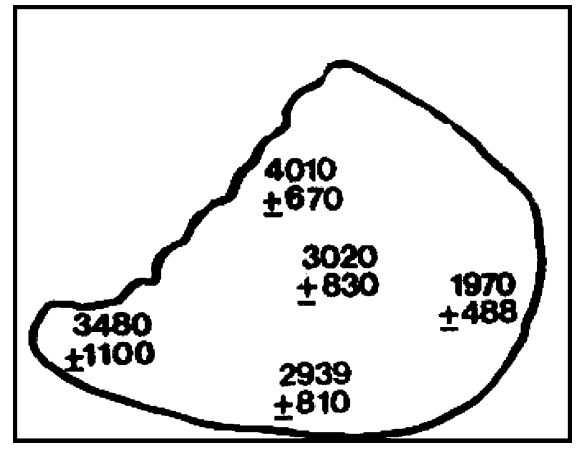

Fig. 2 Activity of the enzyme cathepsin D in five areas of the human septal cartilage. The enzymatic activity is expressed in $\mathrm{mg}$ tyrosine generated per $60 \mathrm{~min}$ per $0.1 \mathrm{mg}$ septal cartilage (adopted from ref. [2]).

sagging and widening of the nasal pyramid as well as impaired breathing (Fig. 3a and b). The long-term effect of the septum destruction is the disturbance of growth, form and function of the nose [6].

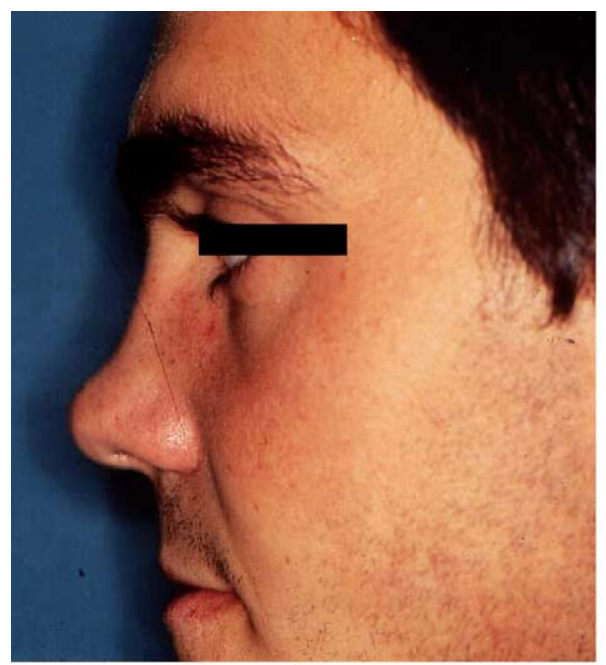

(A)

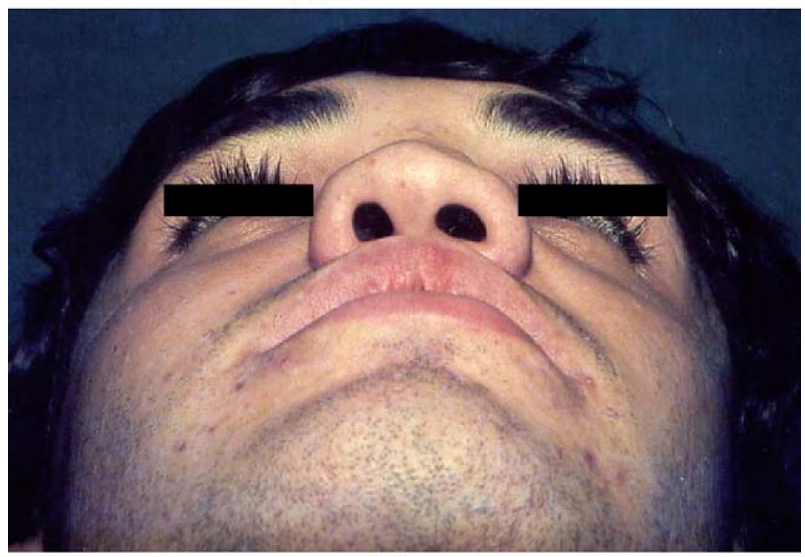

(B)

Fig. 3 (a) and (b) Patient with typical nasal deformities due to absence of septal cartilage (saddle nose). 


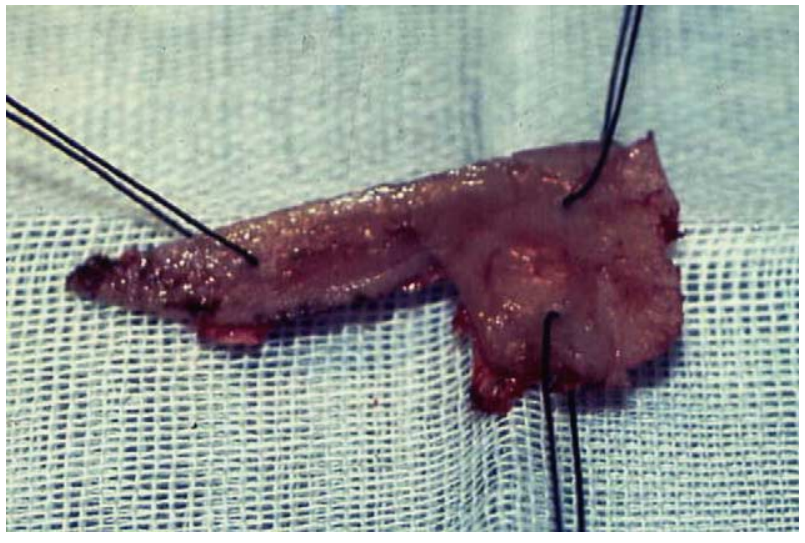

Fig. 4 Homologous L-shaped cartilage from the cartilage bank used for septal graft.

\section{Materials and methods}

Seven patients, aged 3-12 years, were treated for nasal abscess in the period from 1987 to 2000 (ratio male:female, 6:1). All patients had a history of nasal trauma. The diagnosis was made 1-15 days (mean, 8 days) after the episode of trauma. Nasal obstruction, fever and epistaxis were the common presenting symptoms, which, in three cases, were associated with fractures of the nasal bones and deformity of the nasal pyramid.

All patients underwent drainage of the abscess and immediate implantation of the cartilage; a reduction was performed in the cases of nasal bone fractures.

Homologous cartilage from the cartilage bank was used on three patients prior to 1990 (Fig. 4); the mosaic technique, consisting in the reimplantation of multiple small septal pieces in to the septal space fixed together by fibrin glue, was performed on four patient using residual septal cartilage [7]. All patients received packing with Merocel ${ }^{\circledR}$ or gauze which was removed in 3 days. Only two patients required additional packing.

\section{Results}

No complications were observed in the immediate follow-up period. No saddle deformity nor retraction of the columella were observed at six month follow-up and the functional results were satisfactory in all patients. The long-term results observed at 5 and 10 years follow-up periods consisted in a normal development of the face and nasal pyramid (Fig. 5a and b); a sub-stenosis deviation of the nasal septum was observed in four patients (Fig. 6a and $b$ ).

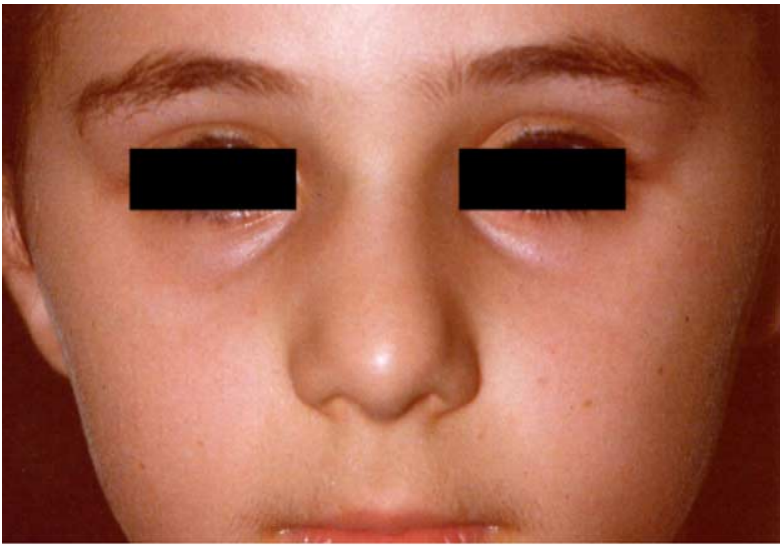

(a)

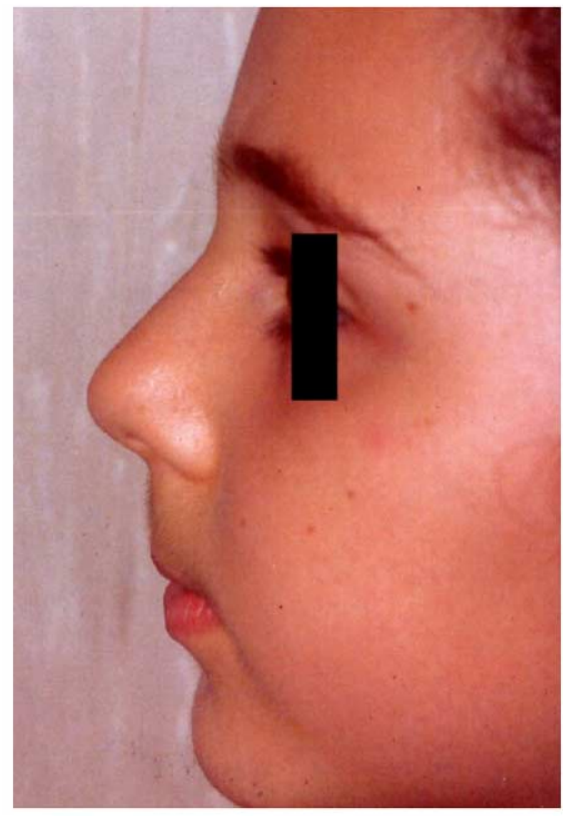

(b)

Fig. 5 (a) and (b) The same patient as Fig. 1 (follow-up at the age of 5): regular development of the nasal pyramid (the treatment consisted of drainage of the abscess and mosaicplasty of the nasal septum).

\section{Discussion}

The management of nasal septal abscess should consider short-term effects, nasal obstruction, saddle nose, retraction of the columella, without neglecting long-term ones such as the disturbance of growth and form of the nose and midface region [6]. The two main treatments published in literature are the surgical drainage $[1,3-5,8,9,12]$ and the drainage and immediate reconstruction of the destroyed septal cartilage $[2,6,7,10,11]$ Cottle has proposed the implantation of nasal septum since 1953 recommending the treatment within 8-12 weeks from the abscess when the infection 


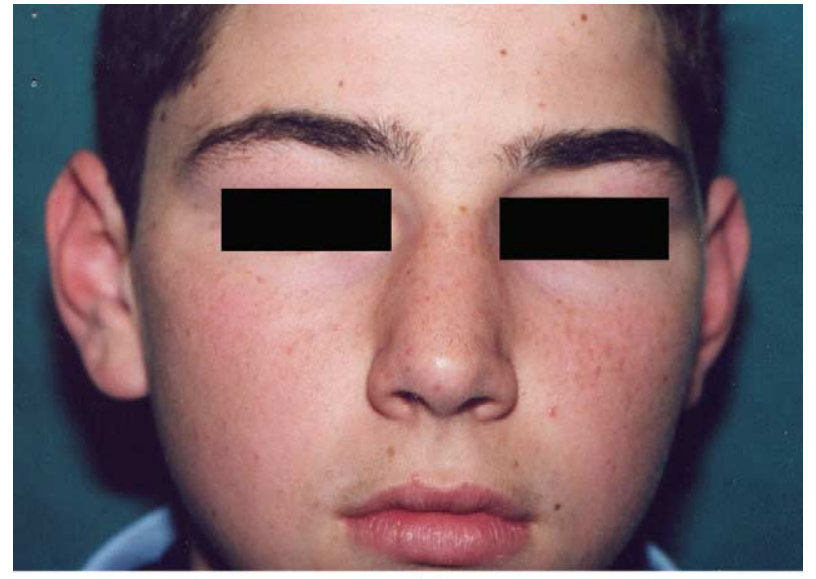

(a)

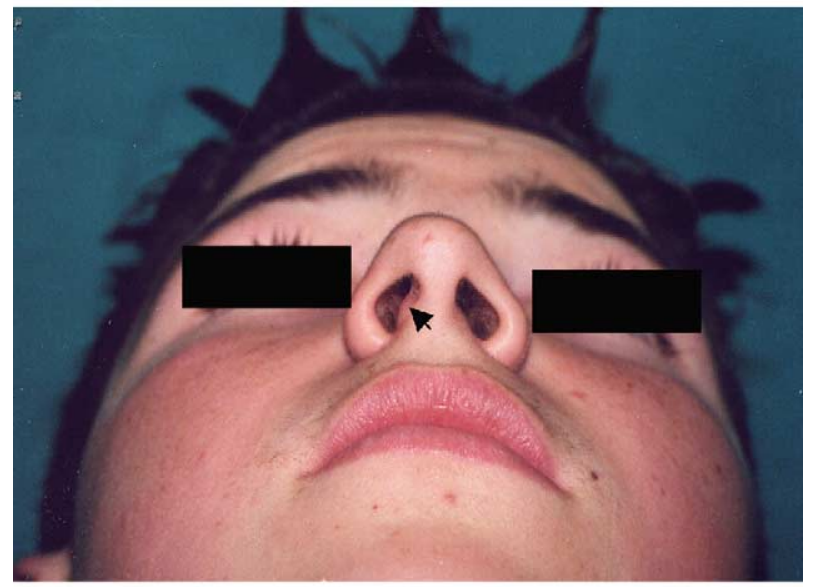

(b)

Fig. 6 (a) and (b) A patient aged 15 (follow-up at 10 years): harmonic development of the nasal pyramid and of the midface region (the treatment consisted of drainage of the abscess, reconstruction of the cartilaginous septum by grafting of homologous cartilage and reduction of the fracture of the nasal bones). Dislocation of antero-inferior septal cartilage in right vestibule (arrow).

resolution could assure a successful implantation [14]. Afterwards, Huizing e Masing proposed a septal reconstruction in the acute phase of a septal abscess, at the same time as drainage [2]. Various authors $[2,7,10]$ have adopted the latter treatment reporting satisfactory results. Hellmich suggested three possible options for the reconstruction of the destroyed septal infrastructure:

(1) the use of posterior cartilage or bony septum residues to adjust deformities in the anterior septum ("exchange technique ");

(2) the mosaicplasty with small fragments of residual cartilage;

(3) the use of preserved rib cartilage allograft whenever the septal material is not available.

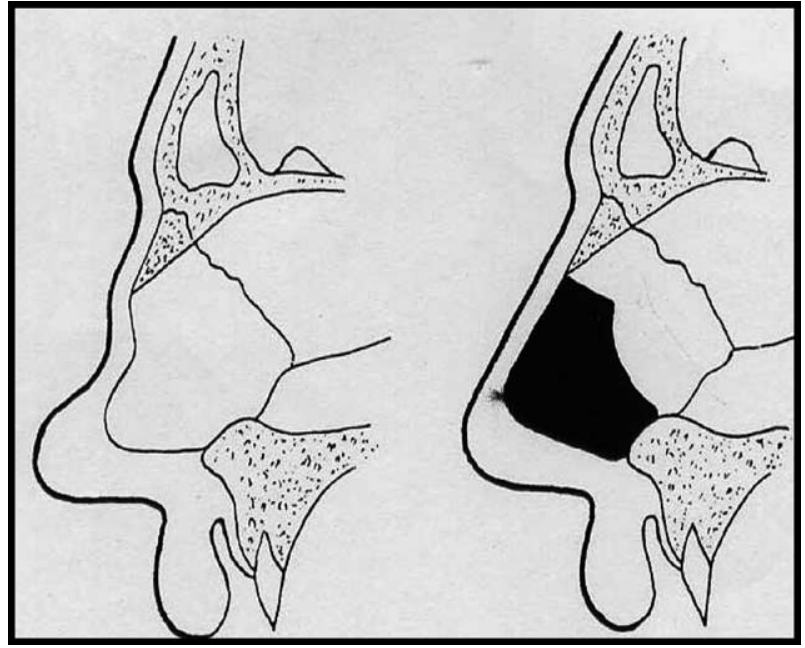

Fig. 7 A boomerang-shaped septal graft (adopted from ref. [7]).

The first and third treatment are unsuitable for children. The former, in fact could destruct the posterior septum and impedes septal growth, while the latter would be too invasive for young patients. Whenever the cartilage fragment is sufficiently wide, Hellmich suggests a "boomerang-shaped" implantation which provides two supports ( $\mathrm{K}$ area and anterior nasal spina) and allows a good development of the midface region [7] (Fig. 7).

\section{Conclusions}

Long-term results have led us to consider the drainage and immediate reconstruction of the nasal septum as a very effective treatment of the septum infected hematoma. It allows a good development of the nasal pyramid and midface region while preventing the short-term effects of this pathology (saddle nose and nasal obstruction) A symptomatic deviation of the septum, as reported in literature, was observed in four cases.

\section{References}

[1] P.S. Ambrus, R.D. Eavey, A.S. Baker, W.R. Wilson, J.H. Kelly, Management of nasal septal abscess, Laryngoscope 91 (1981) 575-582.

[2] W. Pirsig, Historical notes and actual observations on the nasal septal abscess especially in children, Int. J. Ped. Otorhinolaryngol. 8 (1984) 43-54.

[3] C.M. Ginsburg, J.L. Leach, Infected nasal septal hematoma, Ped. Infect. Dis. J. (1995) 14.

[4] M. da Silva, J. Helman, I. Eliachar, H.Z. Joachims, Nasal septal abscess of dental origin, Arch. Otolaryngol. 108 (1982) 380-381. 
[5] J. Viscasillas, S. Viscasillas, P. Claros, Abscesos y hematomas del tabique nasal en la edad pediatrica, Anales ORL Iber-Am. 11 (1984) 419-429.

[6] E.H. Huizing, Long-term results of reconstruction of the septum in the acute phase of a septal abscess in children, Rhinology 22 (1984) 55-63.

[7] S. Hellmich, Reconstruction of the destroyed septal infrastructure, Otolaryngol. Head Neck Surg. 100 (1989) 92-94.

[8] A. Ehrlich, Nasal septal abscess: an unusual complication of nasal trauma, Am. J. Emerg. Med. 11 (1993) 149-150.

[9] P.A. Canty, R.G. Berkowitz, Haematoma and abscess of nasal septum in children, Arch. Otolaryngol. Head Neck Surg. 122 (1996) 1373-1376.

[10] P. Vase, J. Johannessen, Homograft cartilage in the treatment of an abscess in the nasal septum, J. Laryngol. Otol. 95 (1981) 357-359.
[11] L.F. Grymer, C. Bosch, The nasal septum and the development of the midface. A longitudinal study of a pair of monozygotic twins, Rhinology 35 (1997) 6-10.

[12] H. Alvarez, J. Osorio, J.I. De Diego, M.P. Prim, C. De La Torre, J. Gavilan, Sequelae after nasal septum injuries in children, Auris Nasus Larynx 27 (2000) 339342.

[13] I. Brook, Recovery of anaerobic bacteria from a post-traumatic nasal septal abscess. A report of two cases, Ann. Otol. Rhinol. Laryngol. 107 (1998) 959-960.

[14] M.H. Cottle, T.J. Quilty, R.A. Buckingham, Nasal implants in children and in adults; with preliminary note on the use of ox cartilage, Ann. Otol. 62 (1953) 169-175.

[15] A.L. Beck, Abscess of the nasal septum complicating acute ethmoiditis, Arch. Otolaryngol. 42 (1945) 275279.

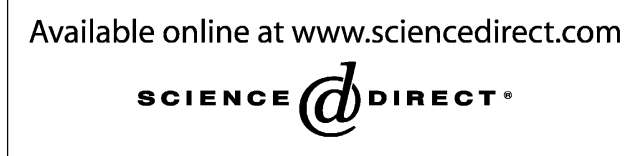

